\title{
Development of an artificial neural network processing technique for the analysis of damage evolution in pultruded composites with acoustic emission.
}

\author{
Davide Crivellia, Mario Guagliano*a, Alberto Monici ${ }^{b}$ \\ a Politecnico di Milano, Department of Mechanical Engineering, via La Masa 1, 20156 Milano, Italy \\ davide.crivelli@mail.polimi.it \\ mario.guagliano@polimi.it, +39 0223998206 \\ ${ }^{b}$ E.T.S Sistemi Industriali srl, Via Olivetti, 2 - 20041 Agrate Brianza (MI) \\ a.monici@etssistemi.it
}

\begin{abstract}
Acoustic Emission (AE) is a promising technique for the damage detection and the real-time structural monitoring of composite lightweight structures; however data interpretation and discrimination among failure modes from AE data is difficult to be carried out without proper data processing techniques. In this paper, a neural-network based classification of $A E$ signals from tensile tests of pultruded glass-fiber specimens is proposed. A self-organizing map is trained with AE data from one specimen; then the map is clustered with the k-means algorithm. The optimal number of clusters is chosen by a voting procedure that takes into account a number of quality indexes; then the clustered neural network is used to classify AE data from other specimen. Results have shown that the classifier built from a smooth specimen was able to correctly classify other specimens with the same and with a different material layup, and is capable of recognizing signals from notched specimens, thus providing interesting and encouraging indications in view of the application on real structures.
\end{abstract}

Keywords: A. Glass fibres; C. Damage mechanics; D. Acoustic emission; E. Pultrusion; Neural Networks

\section{Introduction}

Composite materials are being increasingly used for the production of lightweight structures, in order to meet the ever stricter weight constraints while maintaining adequate safety levels required. In particular, these materials are being recently taken into account both for lightweight transportation systems and in the civil engineering field [1]. In this latter case they are proposed both for new buildings and for restorations, where their low weight is an advantage not only for the structure itself, but also regarding transport and installation costs.

Anyway, the requirements of composites in civil constructions are very different from the ones of light transport systems. This is due to the much more large amount of material needed in the civil constructions, that makes necessary to use cheaper materials with respect to transport systems like aircrafts, where the

${ }^{*}$ corresponding author 
need to reduce the weight in order to increase the transportable load and to reduce the fuel consumption, makes the mechanical performance of the material the most important and desired attribute of the composites.

Focusing the attention on civil engineering, the pultrusion process (deriving from pull- and -extrusion) is particularly attractive. It allows to create structural profiles of uniform section and of virtually any length, with an economic and highly automated production technique [2], since the process itself involves the extrusion of the matrix and the traction of glass fibers. That makes possible to correctly align the fibers before the matrix is polymerized.

Good candidates for the pultrusion process are GFRP (glass-fiber reinforced polymers), thanks to their lower cost with respect to carbon fiber.

Anyway, like for most of polymer matrix composites, the use of these materials is somehow limited by the development of sudden damage under load. As a consequence, the development of damage tolerant design procedures, like the ones used for metallic materials, is more difficult.

With reference to polymer composites the ability to develop such kind design approaches seems to be strictly related to the development of experimental techniques able to monitor the behavior of the material and to provide a real-time feedback about the damage under development.

Research on pultruded materials should also aim to build a precise structural health monitoring real-time system in order to widen the knowledge of their complex and interacting damage modes and thereby their use. Some authors suggest an interaction between fiber and matrix degradation, and a phase-like damage development characterized by a degradation of stiffness and load bearing capacity. Fatigue damage is thought to be dominated by fiber fracture, matrix degradation and fiber-matrix interface degradation [3], [4]. A previous research of the authors of the present paper has also shown that it is particularly challenging to assess the damage modes with traditional destructive techniques, like scanning electron microscope (SEM) imaging and computerized tomography (CT) scanning [5], [6].

Bearing in mind the previous considerations and results, the development of an experimental technique able to monitor the progression of damage under load and to distinguish the different damage modes in their early phase could provide a useful tool for increasing the knowledge of the failure modes of these materials under different load conditions, with obvious positive reflect on the design procedure development, and for increasing the reliability of constructions made with these materials, when applied in-situ.

Acoustic Emission (AE) is a quite suitable technique for this purpose [7]. It allows to monitor ultrasonic signals (usually between $50 \mathrm{kHz}$ and $1 \mathrm{MHz}$ ) emitted by a material when loaded or subject to irreversible phenomena (damage, degradation, stress corrosion...) [8].

$\mathrm{AE}$ is traditionally used and standardized as a non-destructive technique to assess the structural integrity of metallic components (e.g. pressure vessels and pipelines), but recently it has increasingly spread over as a technique for monitoring composite materials damage [9].

$\mathrm{AE}$ is based on the phenomenon that, during damage, energy is partly released in the form of elastic waves that travel inside the material and reach the surface, where apposite sensors (mainly piezoelectric) can detect the high-frequency vibration and convert it to a voltage-time signal. By using multiple sensors it is also possible, under some material homogeneity assumptions, to calculate the signal source location, and therefore to localize the area where damage is developing. AE also provides a measure of the signal energy, which has some qualitative and partly quantitative relationship with the energy released by the material [10]. 
To avoid the management of big amounts of data, AE signals ("hits") are usually characterized by waveform parameters, the most significant of which are represented in Figure 1. These parameters can be related to the damage modes that generated the signals.

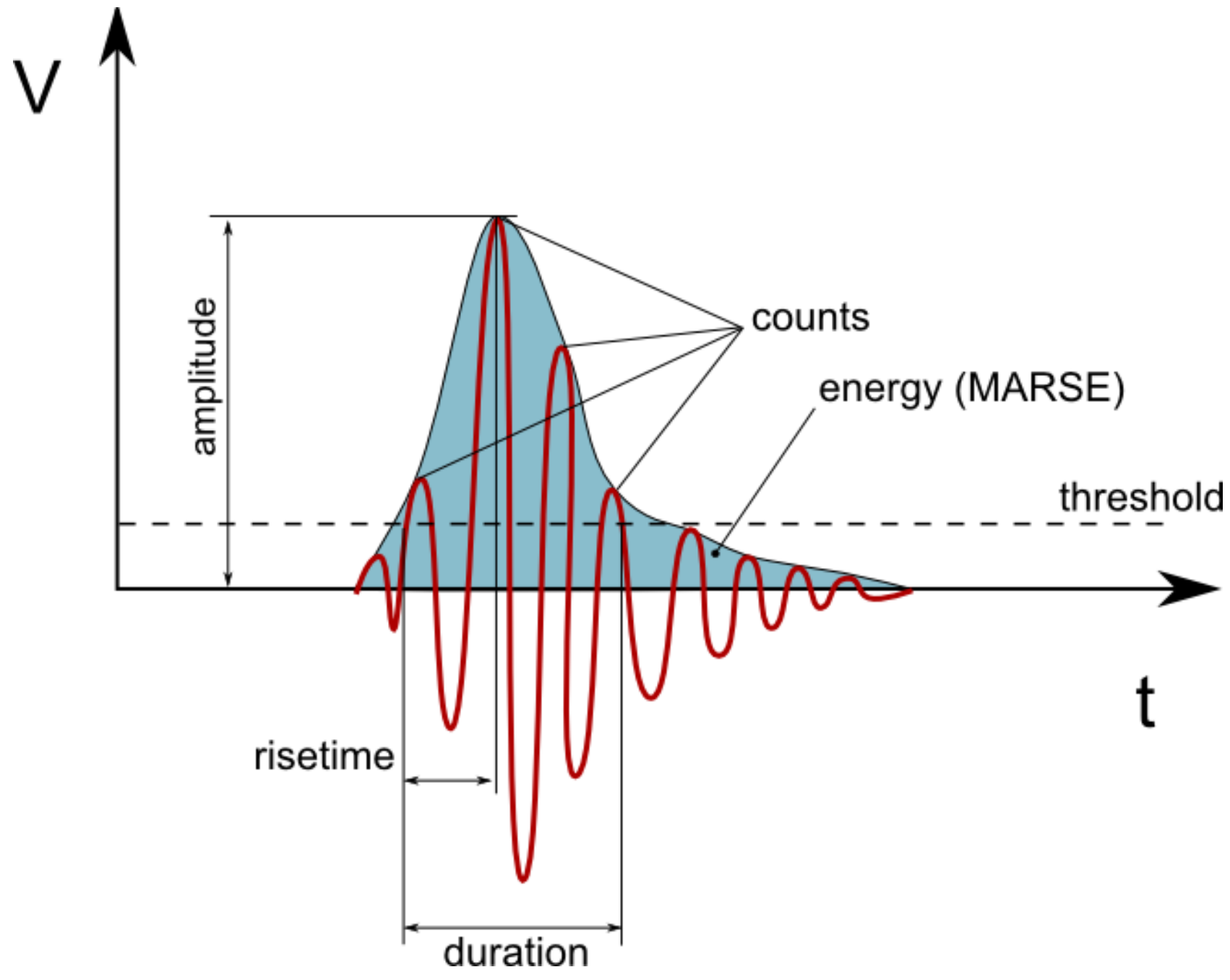

Figure 1: acoustic emission signal typical waveform and parameters

AE on pultruded materials was used [11] for the testing of high voltage power line insulators. A preliminary analysis of $A E$ data was also performed by the authors of this paper [12], even if the results showed the need of a more extensive and exhaustive analysis of the data, that can be performed only by using more advanced signal processing techniques.

In fact, the management of a big quantity of $A E$ data makes often very difficult to perform a traditional data analysis. In addition, a problem has to be solved: the necessity to characterize the type of damage that is responsible of a specific group of signals. Some authors have traditionally proposed the use of some parameters (duration, amplitude and energy) to distinguish among different AE sources [13]; also the frequency content of a signal is considered to be useful [14], but the application of well-defined criteria may be very critical when switching to a different material or even on a different geometry. Kotsikos [15] and Gong et al. [16] have proposed a classification based on amplitude that identifies high-amplitude events as fiber breakage and fiber-matrix friction and pullout, while lower amplitude events are related to matrix microcracking and delamination. 
The so-called classification problem can be solved with different approaches; among them, artificial neural networks (ANN) offer reasonably reliable solutions [17.19].

In this paper, the damage development in glass-fiber pultruded materials is considered and assessed by means of the AE technique. Starting from the results obtained by the authors of this paper in [12], a technique based on a particular architecture of ANN, the so called Self-Organizing Map (SOM) is developed and used in conjunction with the k-means algorithm to elaborate and to separate the different AE signal sources, relating them to the observed phases of damage development. A similar approach that involves unsupervised neural networks and the k-means algorithm has already been successfully applied to the classification AE signals [20]; in this study we explore a way to assess the quality of the classification (and therefore automatically choose the optimal number of clusters) by using different quality indexes and adding frequency information to $A E$ input parameters. Also, in this paper, the feasibility of applying a classifier derived from a single specimen to an entire set of specimens with slightly different layups and with the presence of defects is studied.

After the developed technique is described and applied to the tensile tests executed, the results are critically discussed in view of the application of the technique to structural members.

\section{Experimental setup}

\subsection{Material}

The material used in this study is a pultruded E-glass long fiber reinforced composite. The matrix is a mixture of Leguwal W4 GA and Synolite $0175-\mathrm{N}-1$ resins, which account for $57 \%$ in weight of the material. Additionally, to improve the material behavior in the transverse direction, layers of MAT (randomly oriented long glass fiber) were added (Figure 2). Two configurations were considered: MAT2 (with a top and a bottom MAT layer) and MAT3 (like MAT2 but with an additional MAT layer in the volume, in this case called volumat).

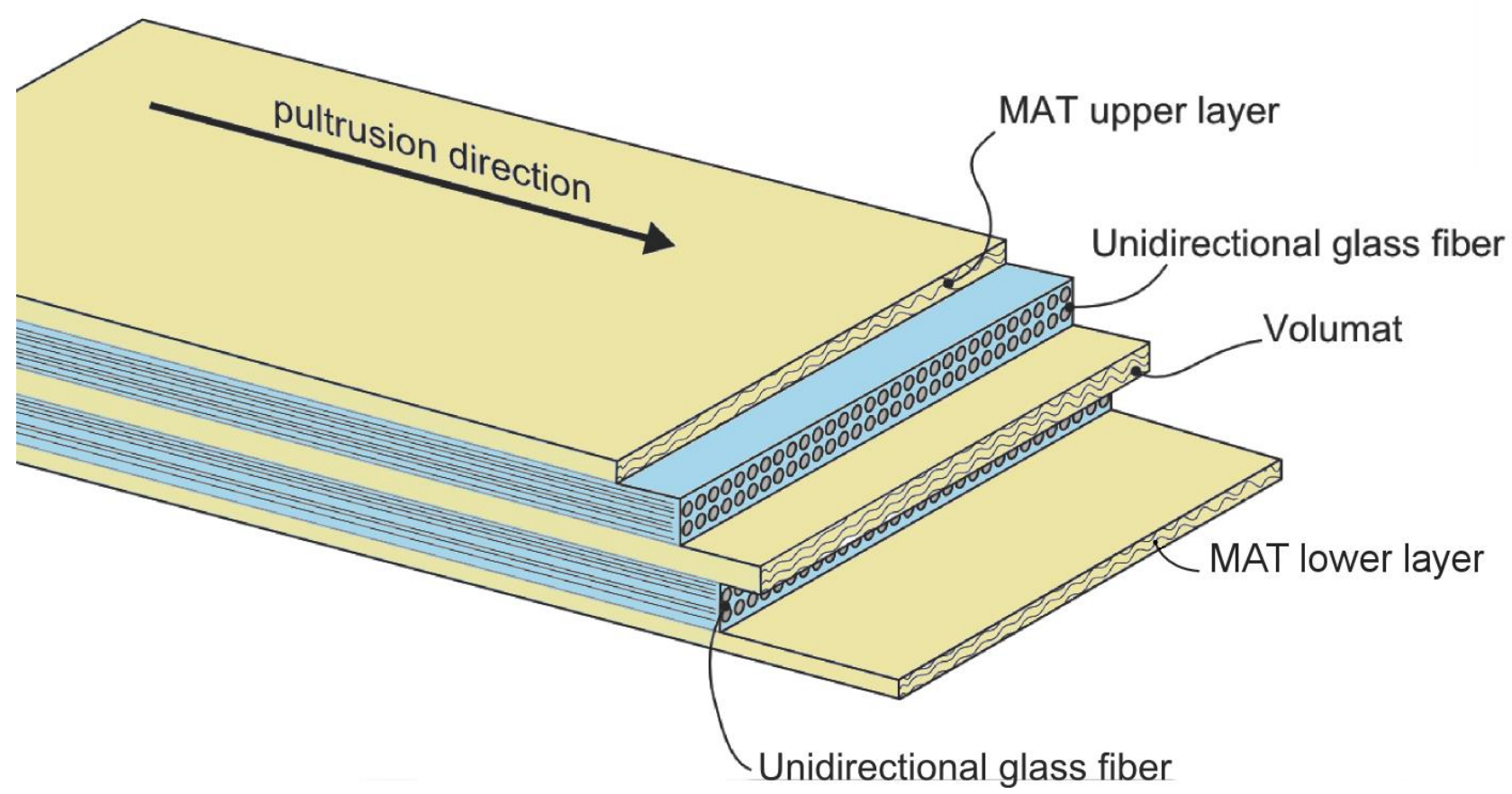

Figure 2: schematic drawing of the MAT 3 structure 
In this experimental work, 16 MAT3 and 20 MAT2 specimens have been analyzed. The experimental procedure has already been described in [12] but is briefly recalled here for clarity and for making easier the understanding of what follows. Specimens were labeled with the layup followed by a number starting from 100; e.g. MAT2_110 represents the $11^{\text {th }}$ MAT2 specimen.

Specimens have been made from rectangular bars in the dogbone shape proposed by [21], since it shows a good uniformity in damage modes and avoids the use of tabs (Figure 3a). Among them, two randomly chosen specimens (MAT2_117 and MAT3_112) were machined to obtain a notch as in Figure 3b, aiming at evaluating differences in damage modes and in $A E$ response caused by the notch itself
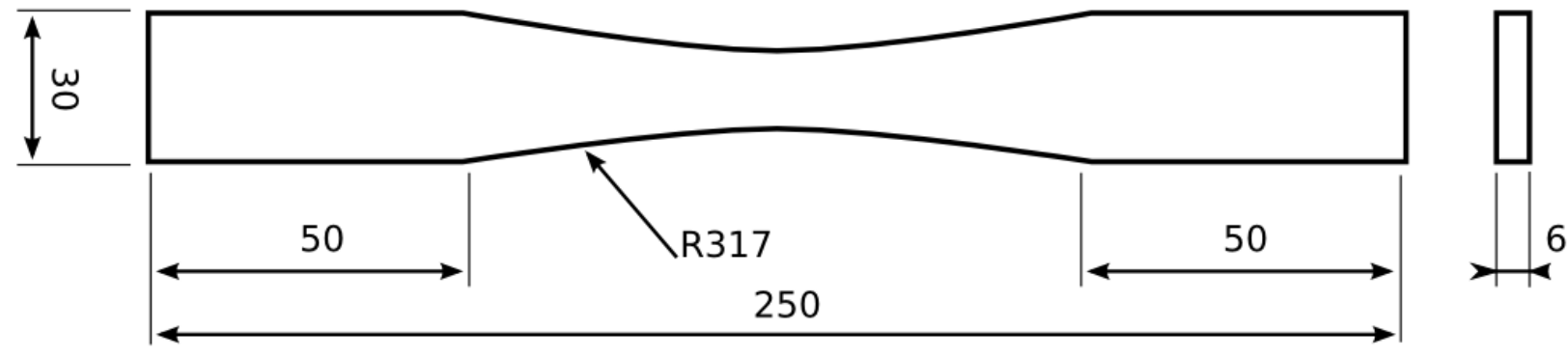

(a)
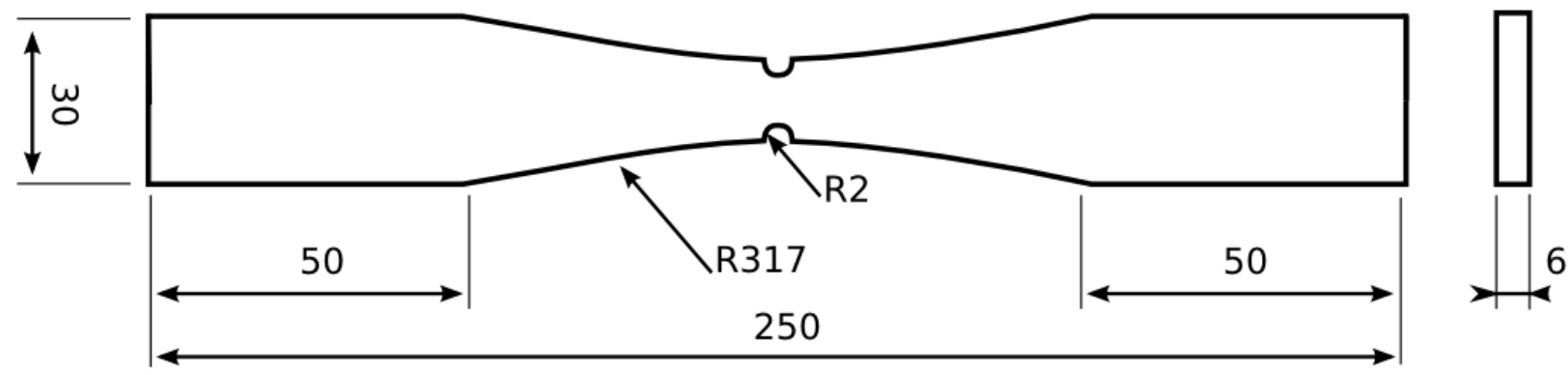

(b)

Figure 3: dogbone-shaped smooth specimen (a) and notched specimen (b) geometry

\subsection{Experimental tests and measurements}

Static tensile tests were carried out on a MTS RT100 electromechanical uniaxial machine, according to ASTM D3039, in displacement control mode. The test speed was set to $2 \mathrm{~mm} / \mathrm{min}$. The specimen elongation was calculated by the testing machine displacement, being the stiffness of the latter higher as compared to the specimen, thus neglecting the error due to the machine deformation; the load was measured through a $100 \mathrm{kN}$ calibrated load cell.

AE signals were recorded with a Vallen AMSY-5 system, connected to a laptop. The load and displacement signals were connected to the $A E$ system, in order to synchronize the AE data with the tensile test data. The system was set up with typical values for short composite samples: duration discretization time (or hit definition time) $=100 \mu \mathrm{s}$; rearm time $=0.2 \mathrm{~ms}$, pretrigger $=40 \mu \mathrm{s}$.

For each specimen, two AE sensors (Vallen VS150M, resonant type) were connected at a distance of $120 \mathrm{~mm}$ using silicone grease; sensors were then attached to preamplifiers with short cables to minimize 
noise pickup, and from the preamplifiers to the AE system with slightly longer cables. The measurement chain is represented in Figure 4.

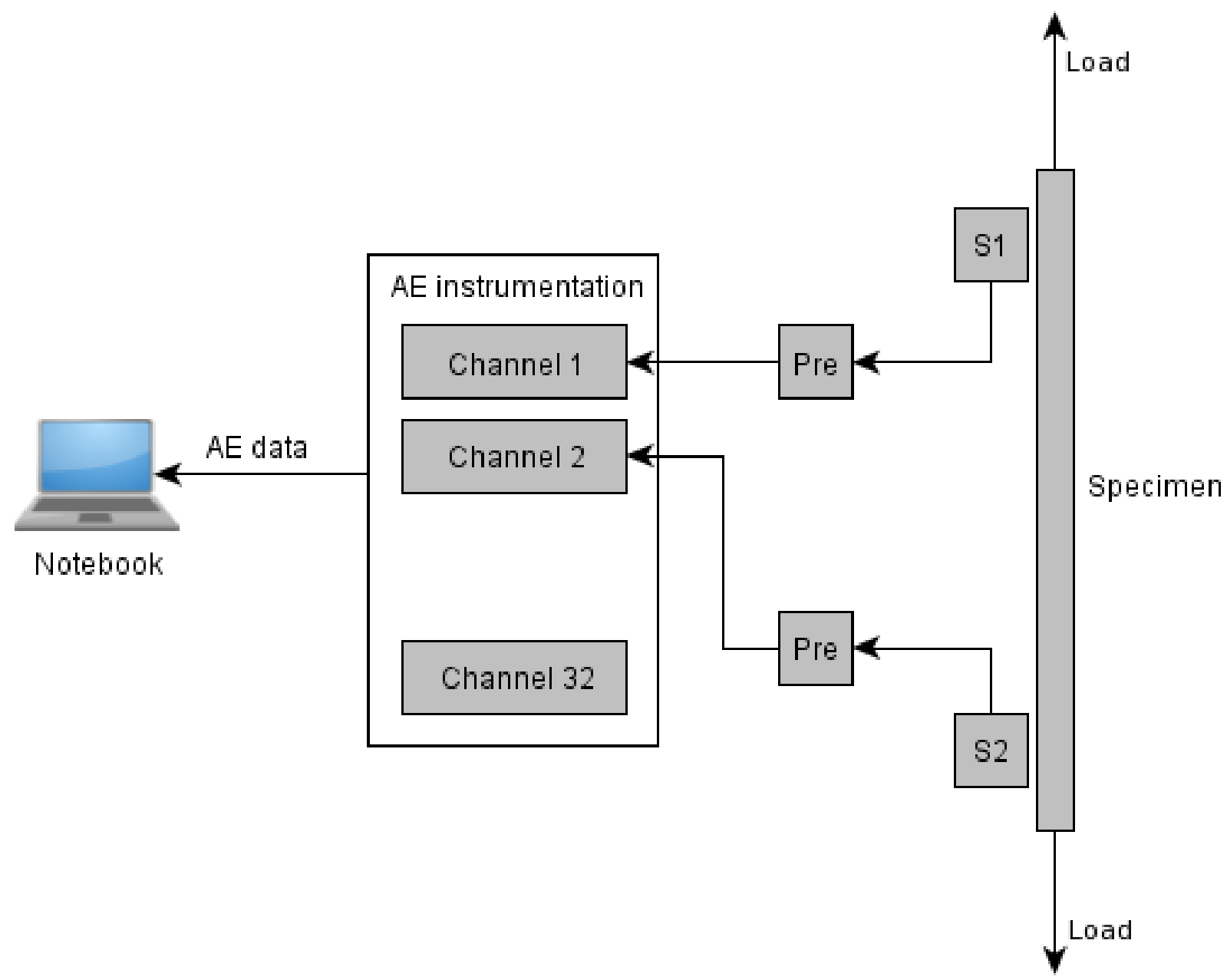

Figure 4: AE measurement chain setup

Before each test, the specimens were mounted in the hydraulic grips of the machine; then 2 minutes of noise without applying any load were acquired to set the noise threshold. For all the tests, a $40 \mathrm{~dB}$ noiserejecting threshold was set.

The system was then set in "pulsing" mode: a sensor emits a signal, and the other receives it; this enables to calculate the wave transmission average speed for the material along the fiber axis. The value was found to be around $3500 \mathrm{~m} / \mathrm{s}$, and this was given as an input to the localization algorithm to get the source position of $A E$ events.

After specimen preparation, tests were started, and AE signals were recorded until the specimen fracture. Tests were stopped when a significant load drop was found, namely $20 \%$ of the maximum load. 


\section{Definition of the classification procedure with Artificial Neural Networks}

As previously mentioned in this work, a particular architecture of ANN, called Self-Organizing Map (SOM), is considered (Figure 5). This kind of network is of the unsupervised type, i.e. it does not require a training in which the outputs are known a-priori but arranges its architecture according to the inputs. This property can be used to classify input vectors based on their relative distance [22]. When a vector is given as an input to the network, a unique "winning neuron" exists (i.e. the neuron with the highest output). The neuron corresponds to the classification of the input vector. Classification of AE data with SOM was applied by [23] with success in identifying different user-generated signals knowing a-priori the source mechanism.

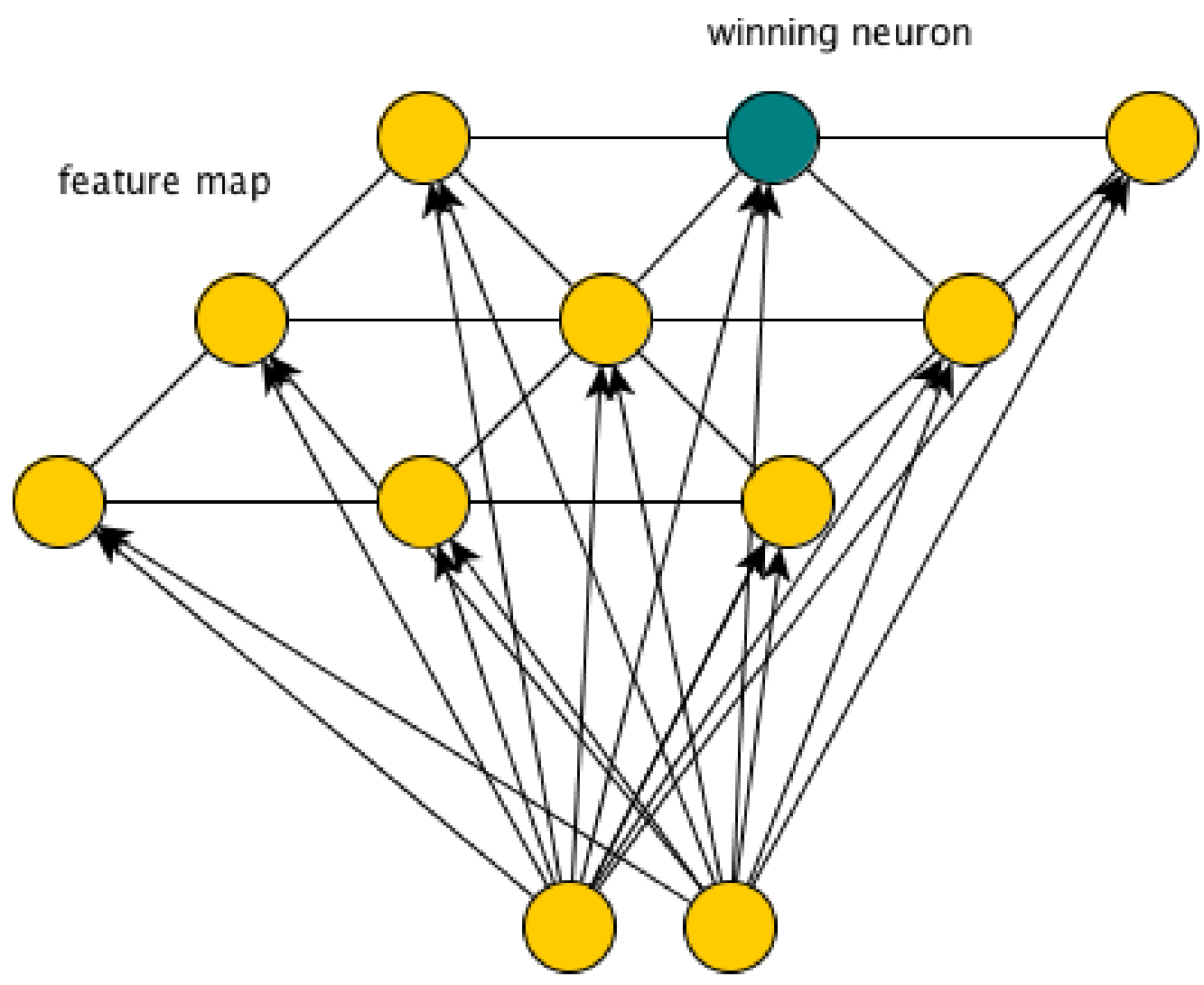

Input layer

Figure 5: Self-Organizing Map representation

Usually, the number of neurons in SOM is much higher than the expected signal clusters. However, looking at the U-matrix (a planar representation of the distances between neurons) allows to observed some degree of separation between neuron groups.

With such framework, it is therefore possible to create a smaller number of clusters, as proposed by [24] with simpler clustering algorithms, like the k-means algorithm. In this way it is possible to classify an input signal into a smaller number of classes. 
The use of the k-means algorithm does not allow to choose a-priori the optimal number of clusters. This is of relevant importance, and some authors [25] have proposed some indexes to measure the quality of a given clusterization. In this work, the approach defined in [26] has been followed and adapted, to use a number of indexes which take into account different parameters of the clusters and to evaluate the best performing number of clusters. This kind of approach is of critical importance, because it is quite straightforward and automatable and could be implemented in any AE-based structural health monitoring system for assessing damage in real-time on a structure under workloads.

All the localized signals, which for every test ranged from 1500 to 2500 , were considered for the data processing. The inputs chosen for the SOM were

- $\quad$ A: max amplitude in $\mathrm{dB}$ (logarithmic scale with a reference voltage of $1 \mathrm{mV}$ at the sensor output);

- $\quad$ D: duration (in $\mu \mathrm{s}$ );

- $\quad$ R: signal rise time (in $\mu$ s);

- $\quad$ CNTS: number of waveform oscillations;

- $\quad E$ : energy of the waveform envelope, in eu $\left(1 \mathrm{eu}=10^{-14} \mathrm{~V}^{2} \mathrm{~s}\right)$;

- $\quad$ FCOG: signal's Fast Fourier Transform center of mass (in kHz);

- $\quad$ FMXA: signal's peak frequency (in $\mathrm{kHz}$ ).

Data points were then normalized between -1 and 1 , and the network was trained with the batch training algorithm.

The clustering procedure proposed in [26] was adapted to use a slightly modified version of the voting scheme proposed in [27]. In particular, after the SOM training, the network U-Matrix was clustered with the k-means algorithm, using a number of classes $c$ ranging from $c_{\min }$ to $c_{\max }$ (in this case from 2 to 17).

To decide the optimal number of clusters, the following three performance indexes were considered and computed:

- Davies-Bouldin [28]

- Silhouette [29]

- Calinski-Harabasz [30]

The use of multiple indexes allows to overcome the limitations and characteristics of every single parameter; for example the Davies-Bouldin index gives small values when clusters are far and compact and is calculated based on similarity between different clusters, whereas the Calinski-Harabasz index is proven useful when the data structure is not known a-priori [31], and the Silhouette index provides information about cluster width and dispersion.

After computing the performance indexes for every considered number of classes $c_{i}$, an aggregate index $L_{i}$ was computed, built so that:

- all the number of cluster are sorted by each performance index separately (best to worst);

- the best cluster number for each index gets $c_{\max }-c_{\min }$ points;

- the $m$-th cluster number gets $c_{\max }-c_{\min }-m$ points; 
- the worst cluster number gets 0 points;

- the points given to each index are then summed for every cluster number.

In this way, if a $c$ number of classes obtains the best value in all indexes, gets 45 points. The best number $c_{\text {best }}$ is then chosen as the number that maximizes the aggregate index.

For this experimentation, a single specimen was used for the SOM training, and then the same network was used to classify other specimens. This was decided due to avoid overtraining of the network, and to assess the robustness of the method when classifying data that the network has never used in the training phase. This decision was also made to determine whether the notched specimens were recognizable by a network trained by AE data from a smooth specimen. A schematic diagram of the process is reported in Figure 6 . For the SOM construction, the SOM Matlab Toolbox [32] was used; the map size considered is 20x16, hexagonal lattice has been used with a sheet structure (i.e. the map border nodes are not connected). The neighborhood function used is a Gaussian; the training phase was done with linear initialization of weights and the batch algorithm, thus using the whole dataset to train the map; the training phase took 3 to 5 iterations. The dataset included between 1500 to 2500 signals depending on the specimen; only localized events were used to help remove noise. 


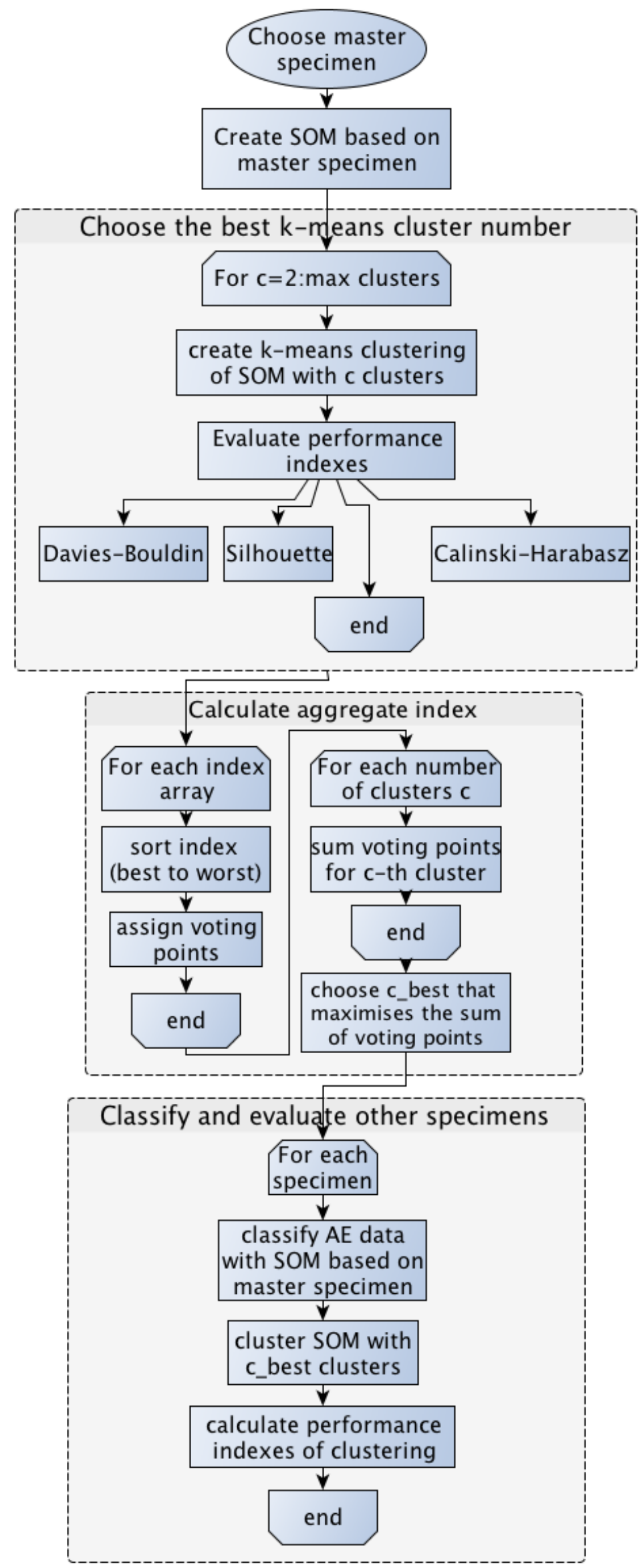

Figure 6: full clustering procedure flowchart 


\section{Results and discussion}

\subsection{Tensile tests}

The results of the tensile tests of the dogbone specimens show the typical behavior of these materials. A first quasi-linear elastic behavior can be noted, followed by irreversible load capacity drops. The first of these drops was considered as limit load, being the irreversible damage unacceptable for any application. Moreover the beginning of this phase coincided to the development of visible defects/damage on the specimen surface.

The two materials (MAT2 and MAT3) showed uniform behavior, with an ultimate tensile strength of 340 MPa (standard deviation equal to $28 \mathrm{MPa}$ ), in accordance with previous testing on the same material [6]. The elastic modulus was found to be different between MAT2 and MAT3 specimens and of $32500 \mathrm{MPa}$ average for MAT2 and 29600 average for MAT3 - this can be easily explained bearing in mind the higher content of long uniaxial fibers for the MAT2 specimen.

The notched specimens had an ultimate tensile test of $272 \mathrm{MPa}$ (MAT3) and $250 \mathrm{MPa}$ (MAT2). The experimental stress concentration factor is found to be below 1.4, which is lower than the theoretical value of 2.5 (estimated from [33]); this is not unexpected since the unidirectional fibers bulk is probably less sensitive and its behavior is far from an isotropic material's.

The main failure modes observed were delamination and fracture of the upper and lower MAT layers upon the first load drop (Figure 7a); by continuing the test further, the failure mode observed was fiber pull-out and explosive delamination (Figure $7 \mathrm{~b}$ ).

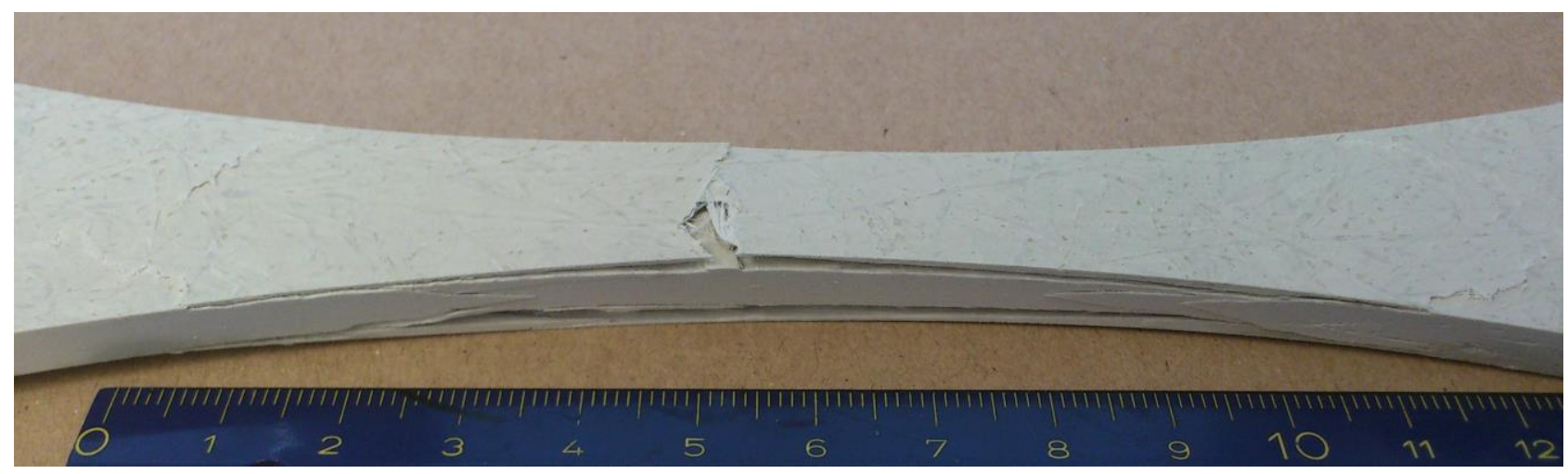

(a)

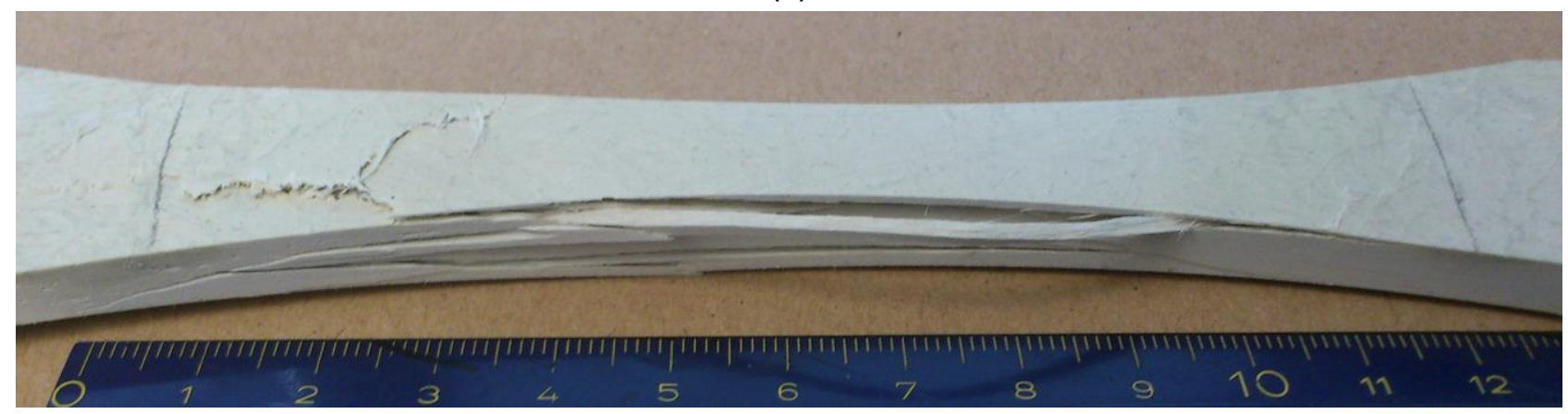

(b)

Figure 7: MAT layer fracture and delamination (a) and explosive delamination (b) 


\subsection{AE signal processing}

$A E$ activity was found to be uniform in the whole dataset; as it can be seen in Figure 8, the cumulate $A E$ activity shows the typical S-shaped curve already seen by [11]. This can be explained by looking at the energy of the signals, which is higher in the last part, thus having fewer events with higher energy towards the final failure.

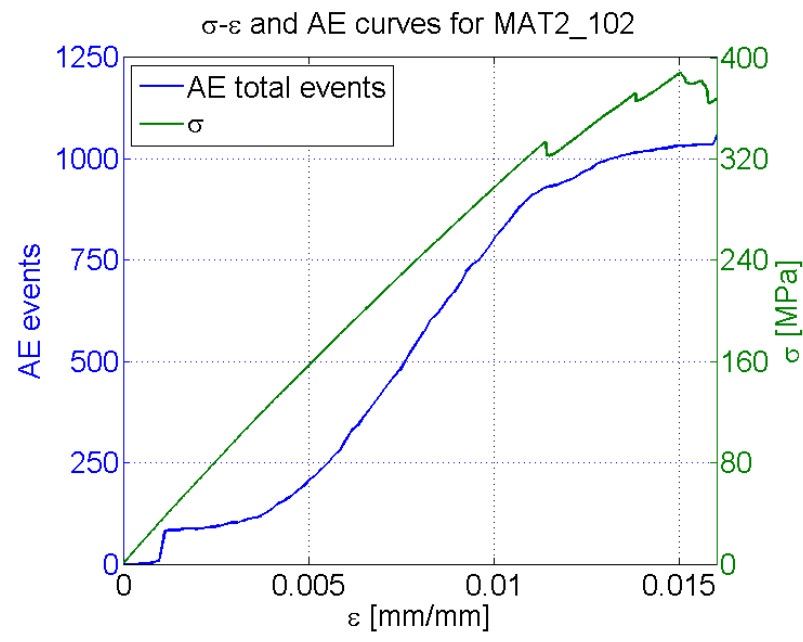

(a)

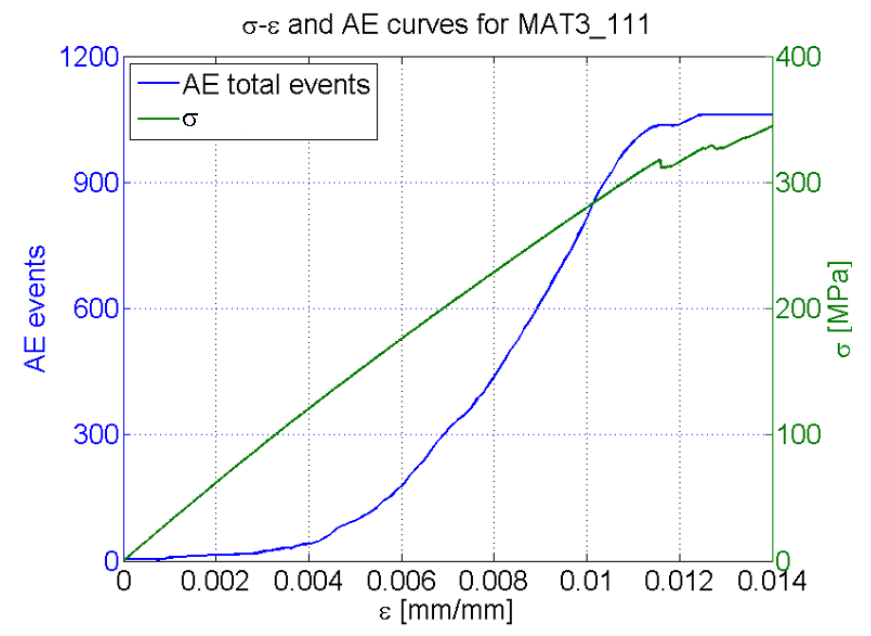

(b)

Figure 8: Acoustic Emission cumulative activity during tests

Data from specimen number MAT3_113 was then used as master specimen to train the SOM: it has to be stressed, however, that changing the master specimen shows no appreciable difference in the process results.

The U-matrix of the trained SOM is reported in Figure 9a. Distinct areas can be clearly seen; however an appropriate algorithm to choose these areas and a measure of the quality of such a classification has to be provided. To perform this evaluation, the k-means algorithm has been applied to the U-matrix (Figure 9b).

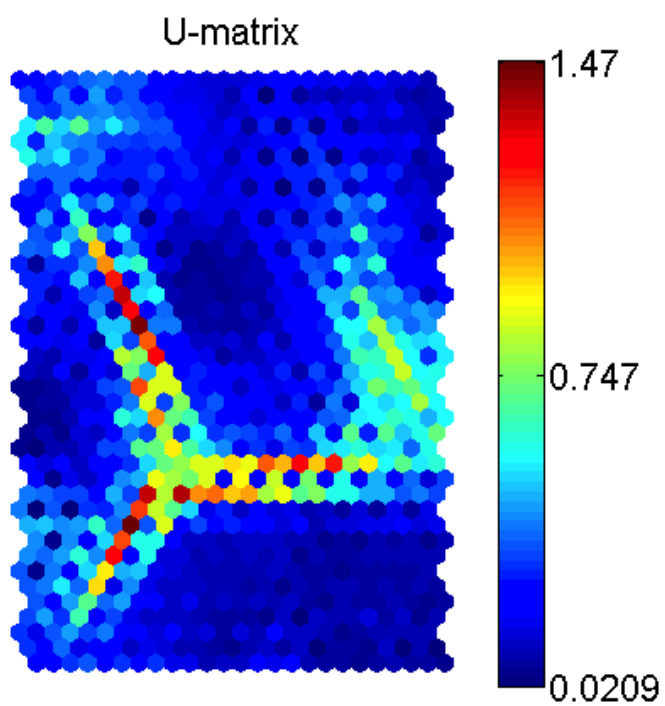

(a)

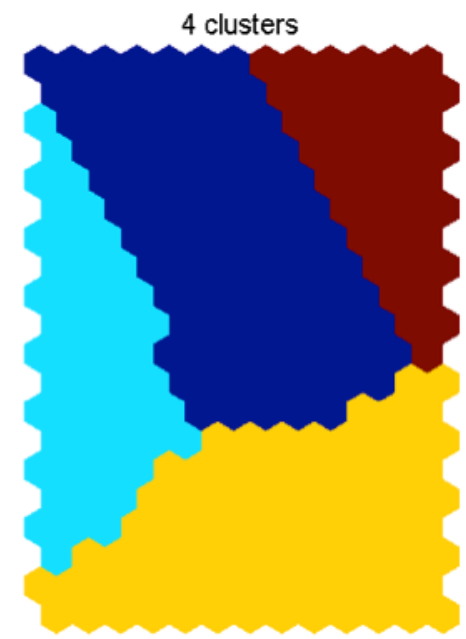

(b)

Figure 9: U-matrix for the SOM trained with master specimen data (a) and clusters (b) 
As previously said, quality indexes and aggregate index for the different cluster sizes have been computed; results are reported in Figure 10. Classification shows that, for this specimen, the Calinski-Harabasz and Davies-Bouldin indexes identify 4 as the best performing cluster number, while for the Silhouette index the best is 3 , immediately followed by 4 . The results of the aggregate index calculation shows that 4 is the most voted clusters number, and therefore it is chosen as $c_{\text {best. }}$ Using a different specimen for classification yields very similar results, identifying always 4 clusters as the optimal solution, but with small variations on the indexes values.

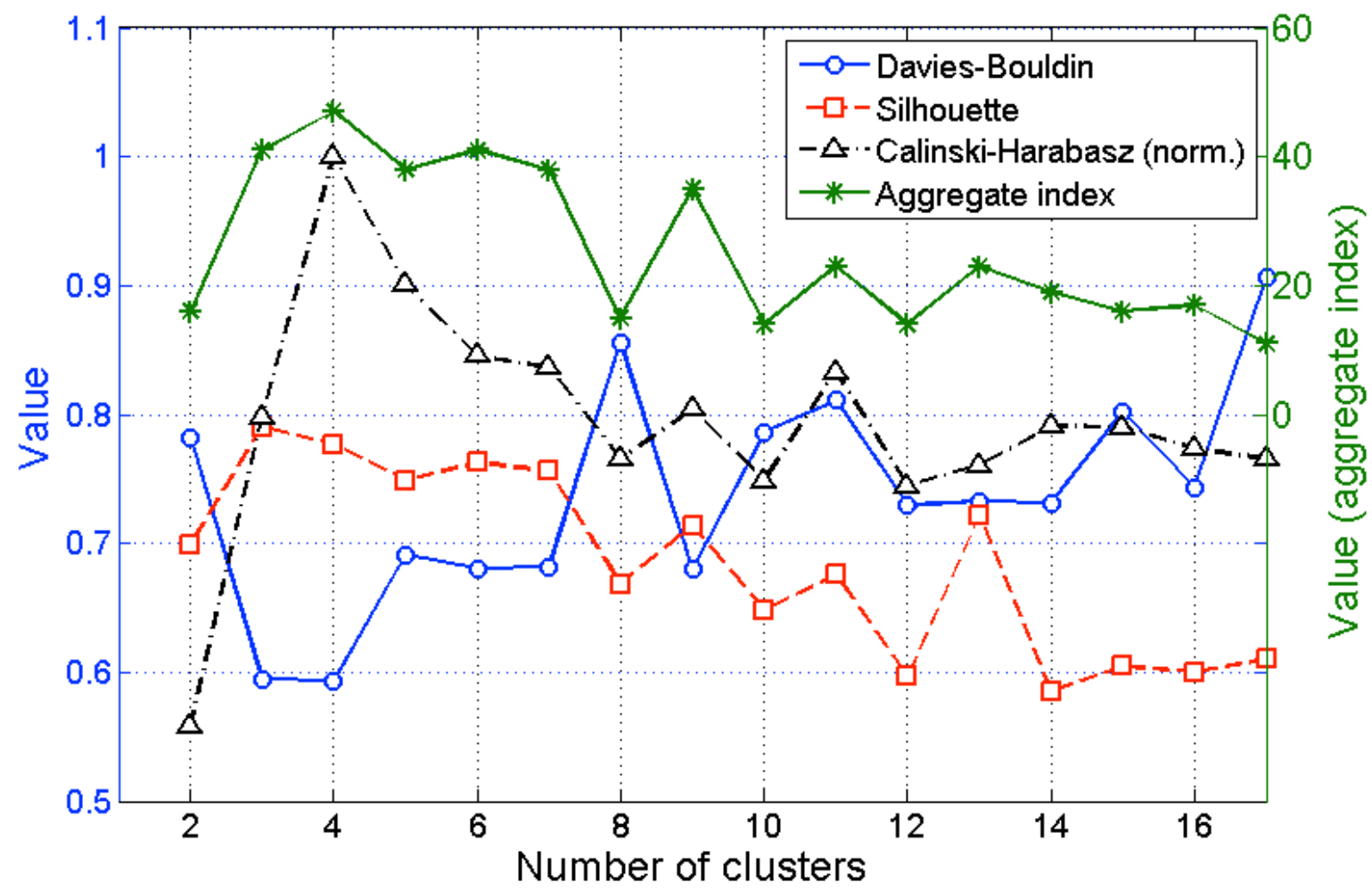

Figure 10: Quality indexes and aggregate index for the master U-matrix clusterization

Remaining specimens have been classified with the master specimen SOM, clustered by the k-means algorithm with $c=4$. As a further verification, the performance indexes have been computed for the other specimens to check if the classification quality changes on different specimens and in particular on the two different material layouts. No appreciable dependence on the material layup or on the specimen number was observed by looking at individual indexes in the two groups.

Waveforms were observed through the separate classes and visually compared to check if the SOM/Kmeans technique was able to separate different signals. A representative signal from each class is shown in Figure 11. 


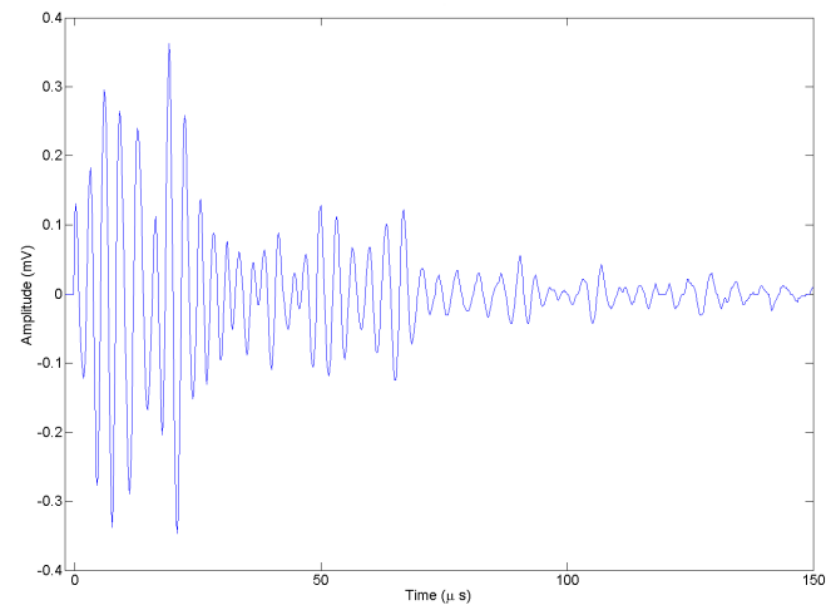

(1)

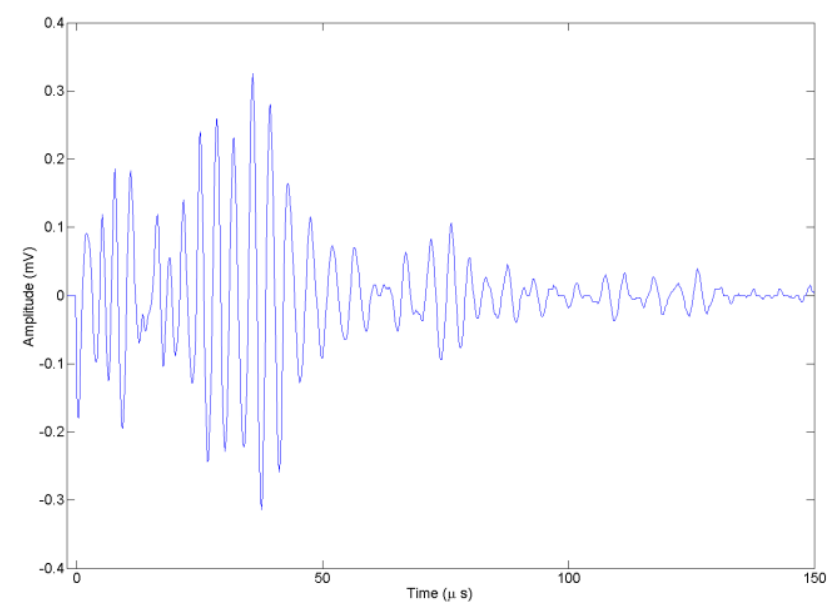

(3)

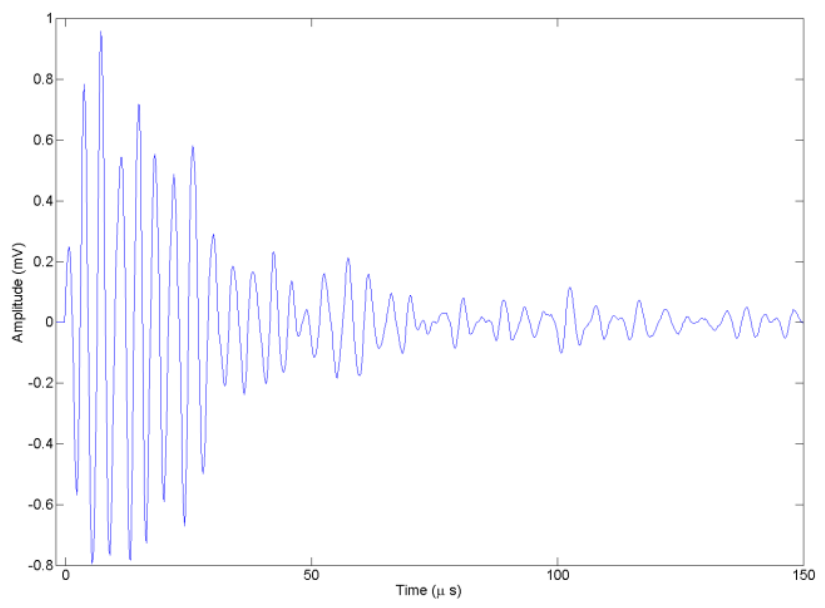

(2)

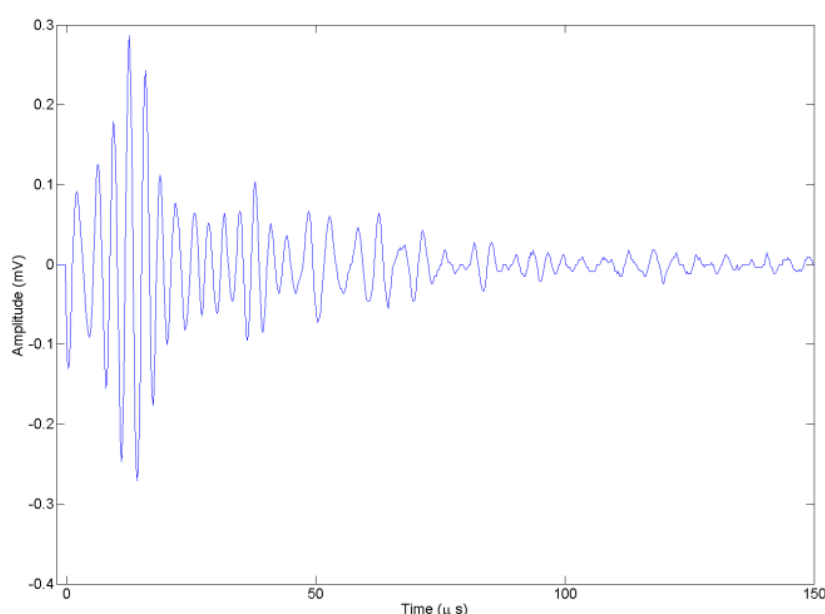

(4)

Figure 11: Examples of waveforms representative of class 1 (a), 2 (b), 3 (c) and 4 (d)

\subsection{Observations on the connection between AE classes and failure modes}

An example of the AE activity curves of the single classes for a smooth specimen and a notched specimen can be seen in Figure 12. For all the other specimens the trend is quite similar. Again we underline that only localized events were considered, and a number of $A E$ events ranging between 1500 and 2500 for every test were recorded. 


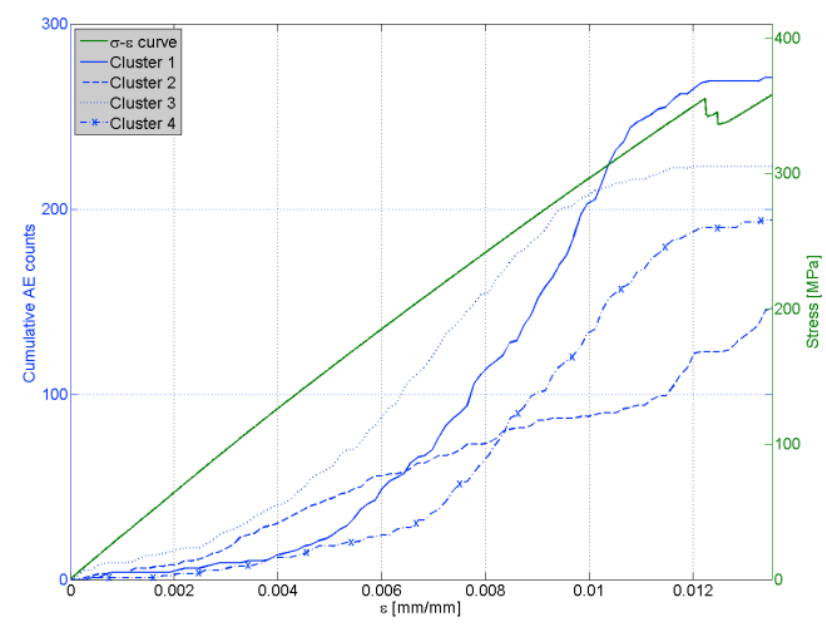

(a)

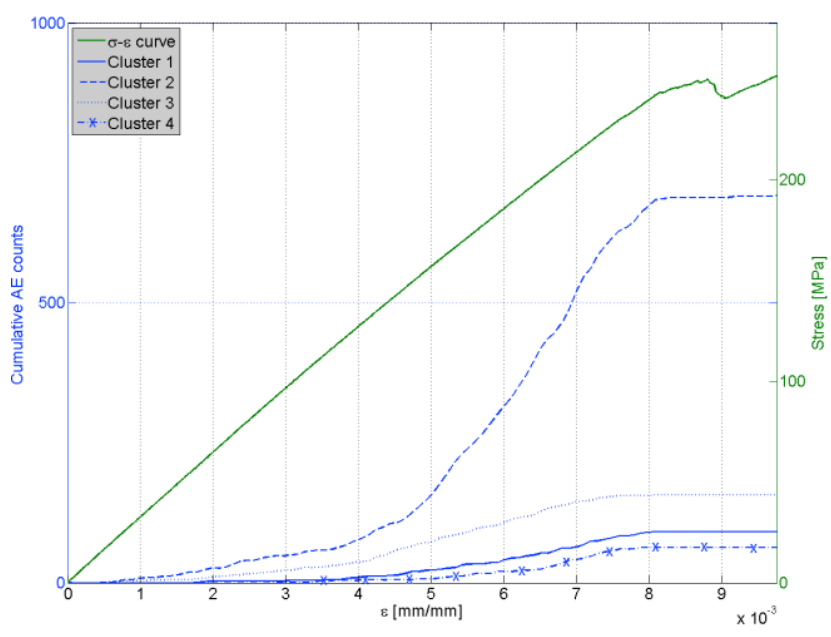

(b)

Figure 12: AE activity of the single clusters for a smooth (a) and a notched (b) specimen

A comparison of all the curves of AE activity and energy for each cluster can be seen in Figure 13. The notched specimens are marked with a thick line. Observation of the comparative curves in all classes shows no significant difference between MAT2 and MAT3 specimens.

Cluster 1 (Figure 13a) shows an S-shaped activity curve that begins at around $40 \%-50 \%$ of final breakage. Energy release is linear in log scale, which means there's an exponential increase of energy release with strain.

Cluster 2 (Figure 13b) shows a more abrupt energy release in the final part; it is also strongly related to higher energy and $A E$ activity in the two notched specimens, which deviate from linearity earlier than $50 \%$ of the test, while energy curves for smooth specimens show an abrupt increase only after $70 \%-80 \%$ of breakage strain.

Cluster 3 (Figure 13c) is again an S-shaped curve that begins at about $20 \%$ of strain, having thus the earliest onset among all clusters; energy release is somehow uniform (decreasing in log scale).

Cluster 4 (Figure 13d) shows a knee between $40 \%$ and $60 \%$ of the final strain, but the position of the slope change is the most spread among specimens. The energy release is again exponential. 
Cluster 2

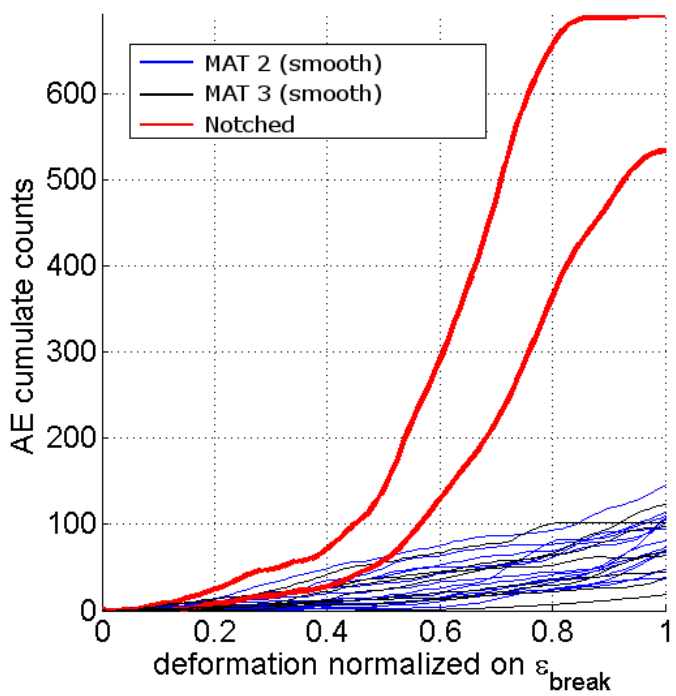

Cluster 2

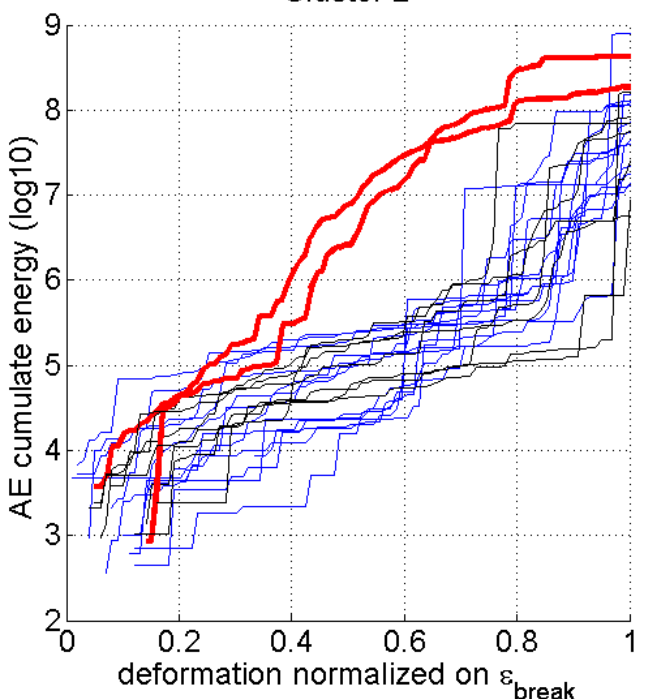

(a)

Cluster 1

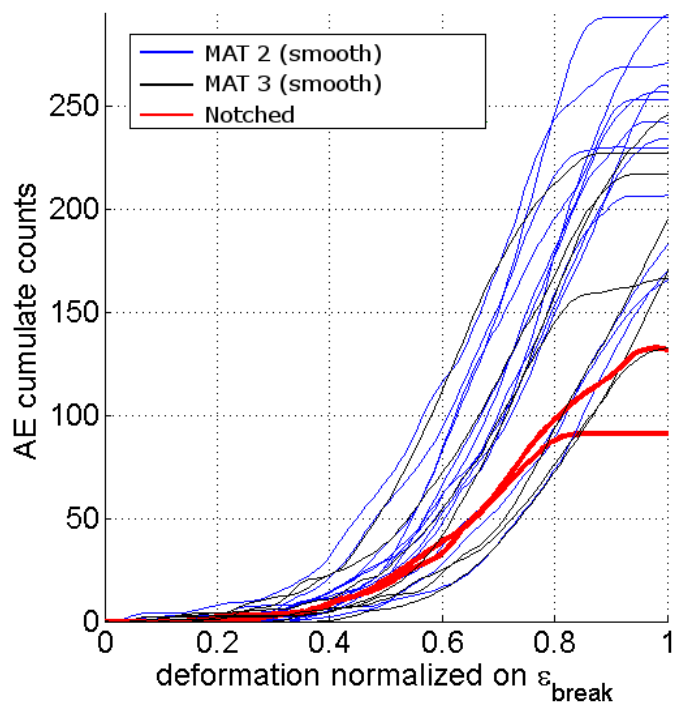

Cluster 1

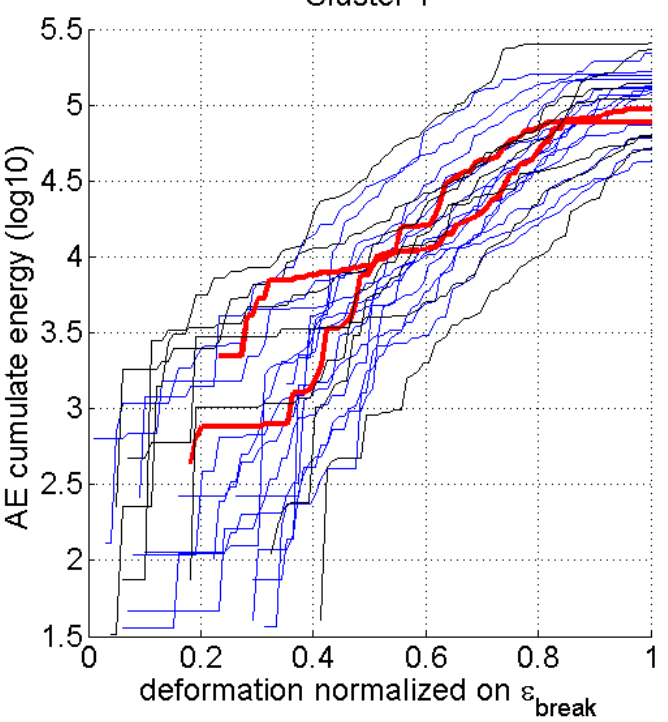

(b) 
Cluster 3

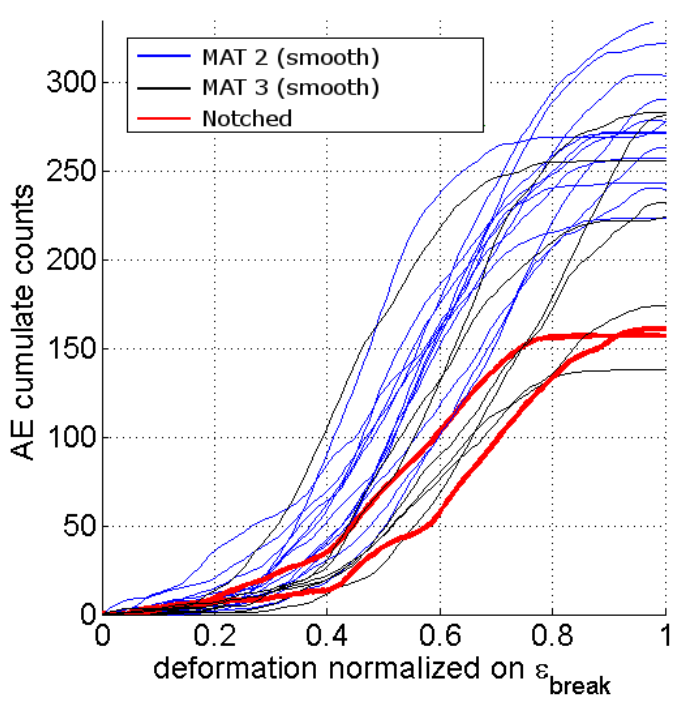

(c)

Cluster 4

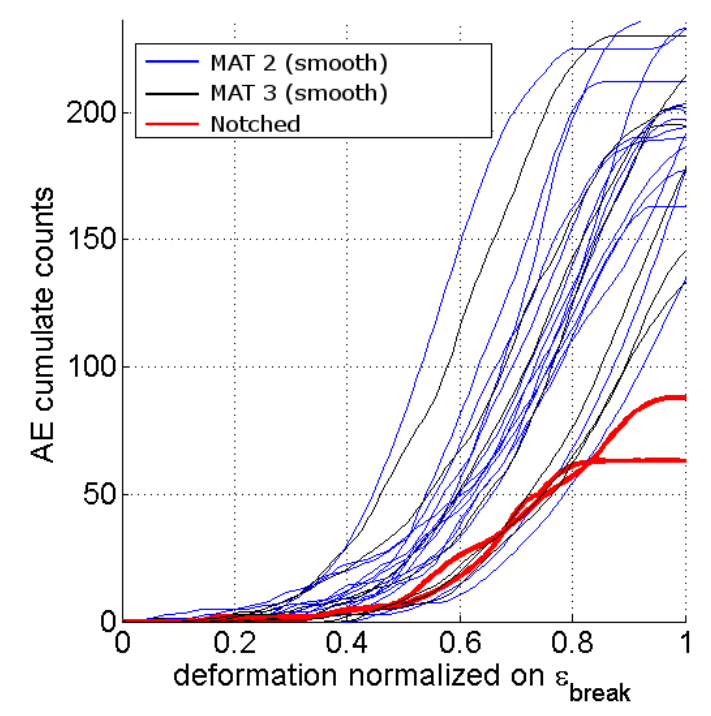

Cluster 3
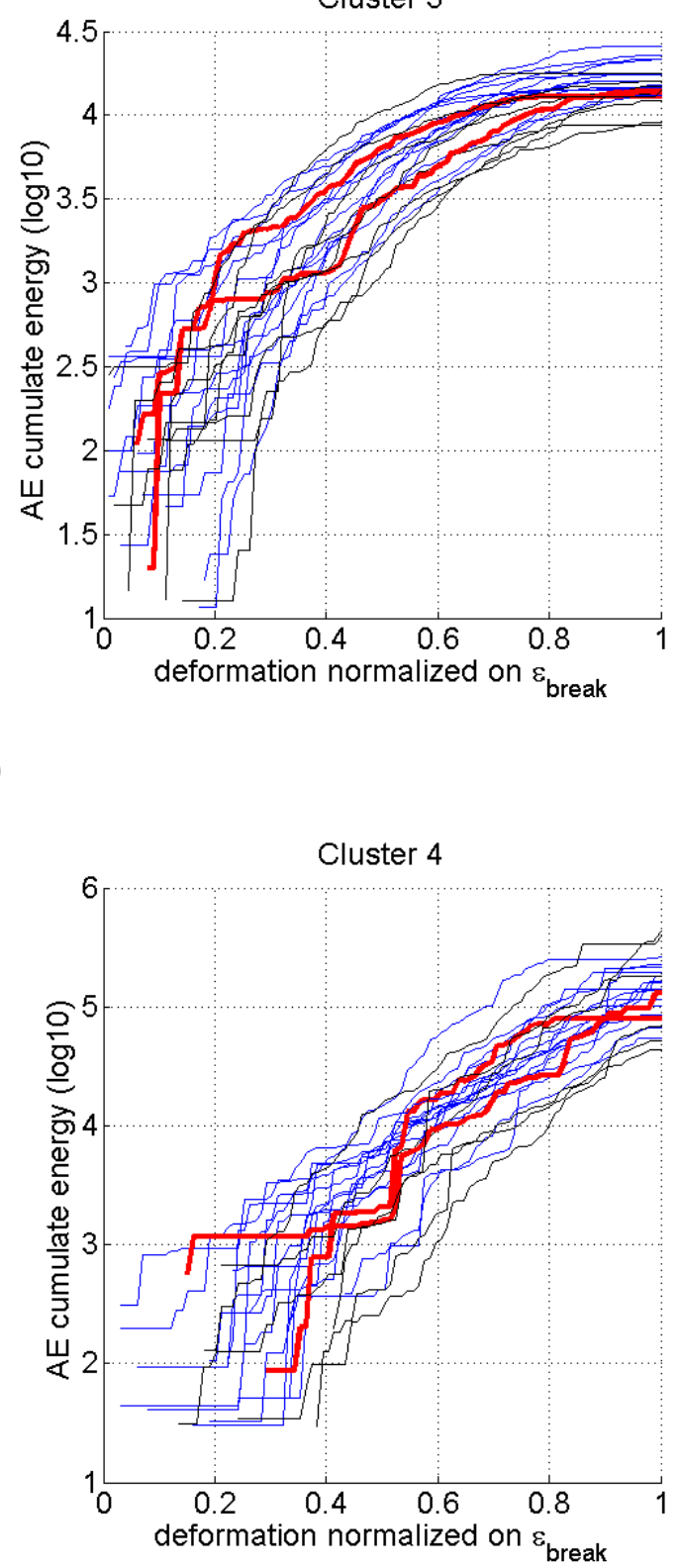

(d)

Figure 13: comparison of AE activity and energy for all specimens: (a) cluster 1, (b) cluster 2, (c) cluster 3, (d) cluster 4

Energy activity of the notched specimens for clusters 1, 3 and 4 show no appreciable difference from smooth specimens, while in the same clusters the AE cumulate counts curves show a slightly lower activity towards the end of the test. AE activity of cluster 3 shows an early onset and is the first active class in every specimen. Clusters 4 and 1 have similar shapes, with cluster 1 being slightly active than cluster 4 . Clusters 1 , 3 and 4 show a S-shaped curve with a linear central part, while cluster 2 is almost linear, with an early begin and an abrupt increase near the limit stress.

Location data of the AE signals along the specimen axis were also computed. Figure 14 shows the energy release (colored scale) during a test (vertical axis) and along the specimen axis (horizontal axis) for a smooth specimen. The same behavior is found in all smooth specimens. Figure 15 shows the energy release for a notched specimen. 

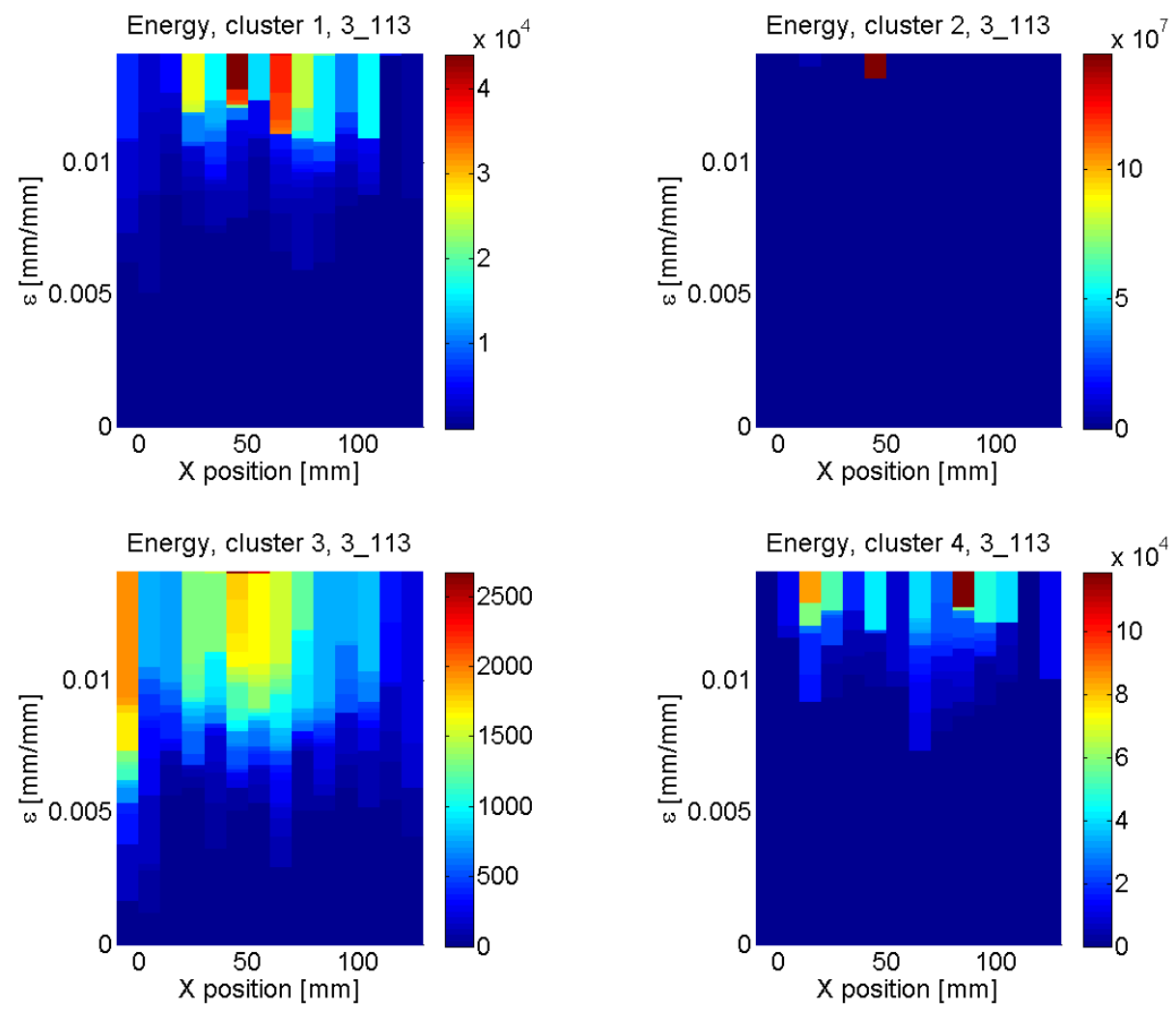

Figure 14: energy release for each cluster by $x$ location, specimen MAT3_113
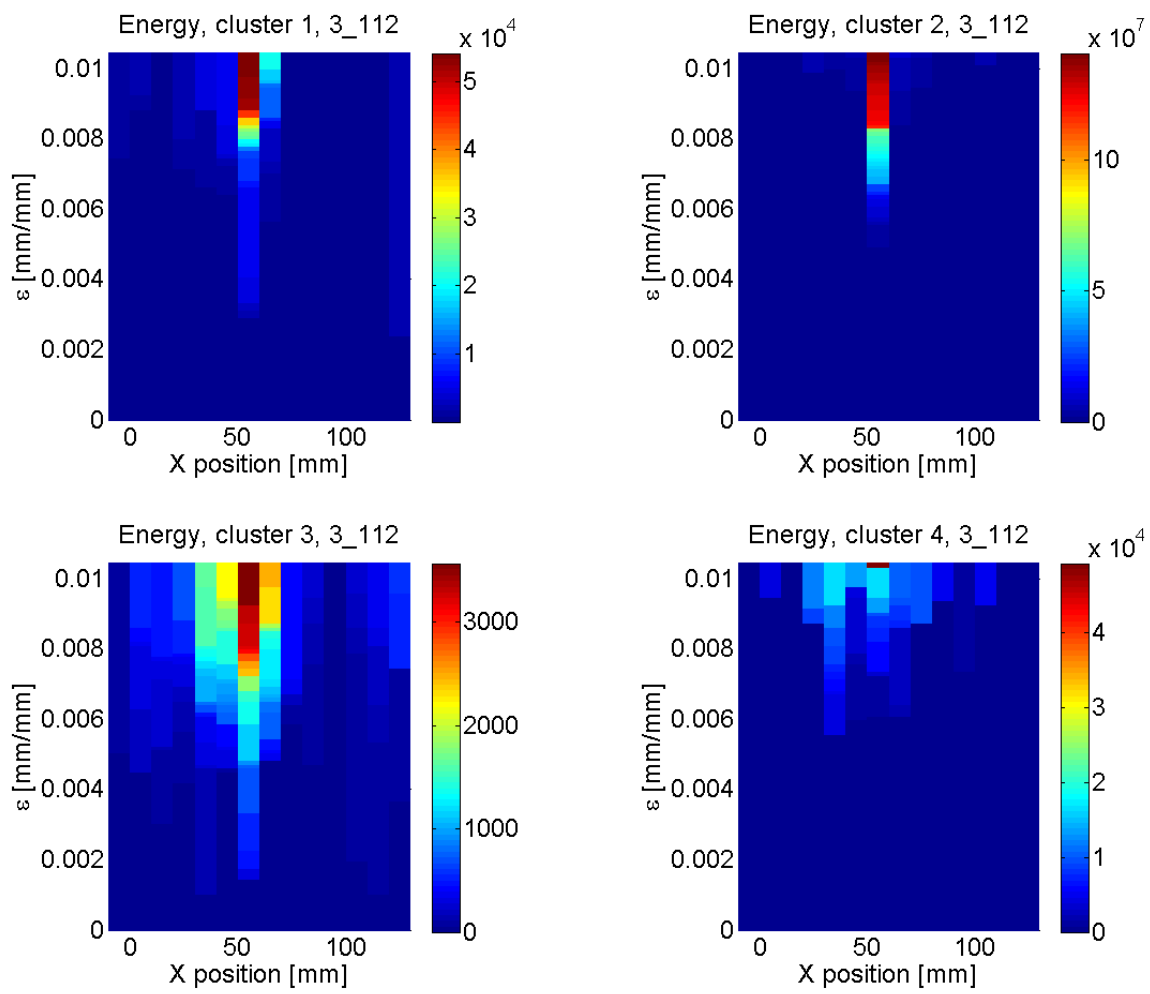

Figure 15: energy release for each cluster by $x$ location, notched specimen MAT3_112 
In particular, cluster 1 shows local high-energy releases around 3/4 of the test; its higher energy release is believed to be connected to the breakage of the top and bottom MAT layers, which is confirmed by visual observation of post-test specimens and location comparison.

Events of cluster 2 show a linear response with sudden energy release towards the end of the test. In the case of the notched specimens the same cluster shows a behavior that is distinctly separable from smooth specimens. In particular this can lead to conclude that events of that class are related to near-end failure modes, such as fiber breakage.

Cluster 3 energy distribution has a uniform energy release that recalls the shape of the specimen, with more energy released in the smaller areas and less energy released in the larger areas; this leads to suppose that events in this cluster are related to average stress phenomena, such as matrix degradation. In the notched specimens the specimen shape is still recalled with an energy release concentration around the notch (Figure 16).
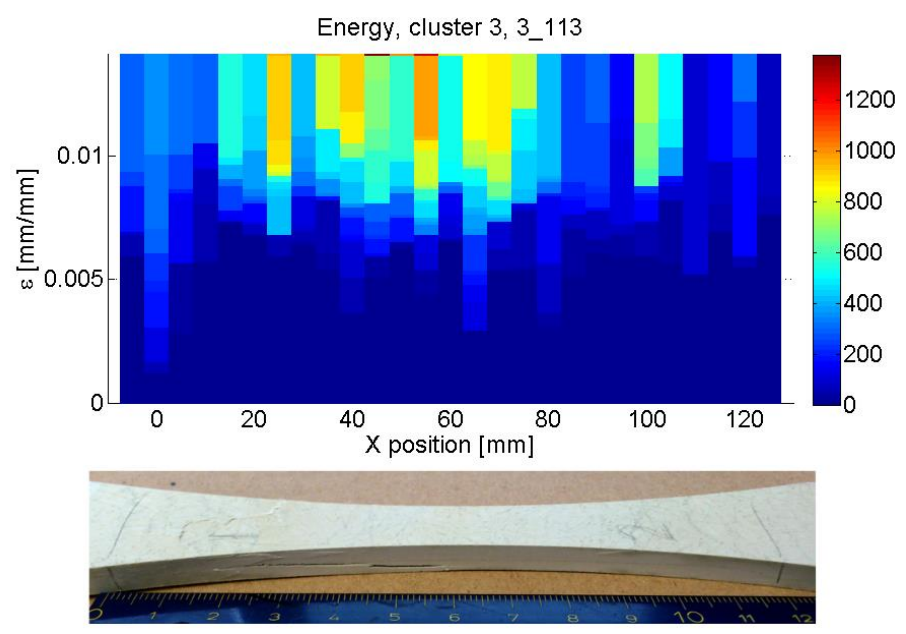

(a)

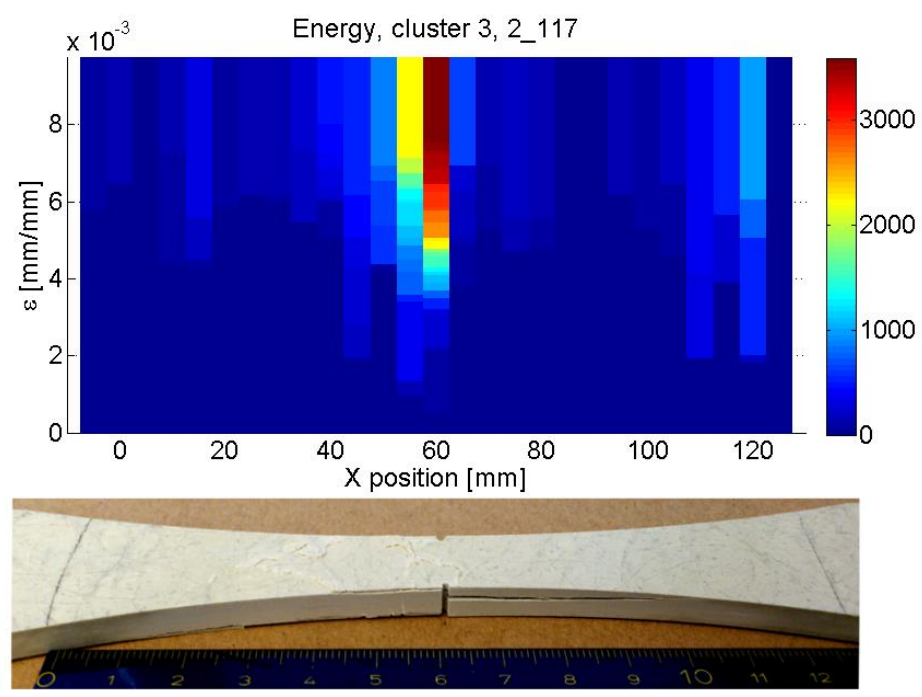

(b)

Figure 16: comparison of AE energy data and smooth (a) and notched (b) specimen damage 
Cluster 4 shows mixed uniform and localized energy release beginning at $2 / 3$ of the test, but has the least uniform behavior among specimens. It is found in some cases linked to the delamination between the MAT layers and the axial fibers layers.

To support these observations, SEM microstructure images were collected from cross-sections in different positions from a notched and a non-notched specimen. Observations from a smooth specimen show that the small cross-section area failure is dominated by matrix cracking (Figure 17a) and in a position far from the center is dominated by MAT-fiber interface fracture and bulk fracture (Figure 17b), while in notched specimens the situation is reversed, with a fiber-matrix debond dominated central area (Figure 17c) and only matrix crack presence in the farther areas (Figure 17d)

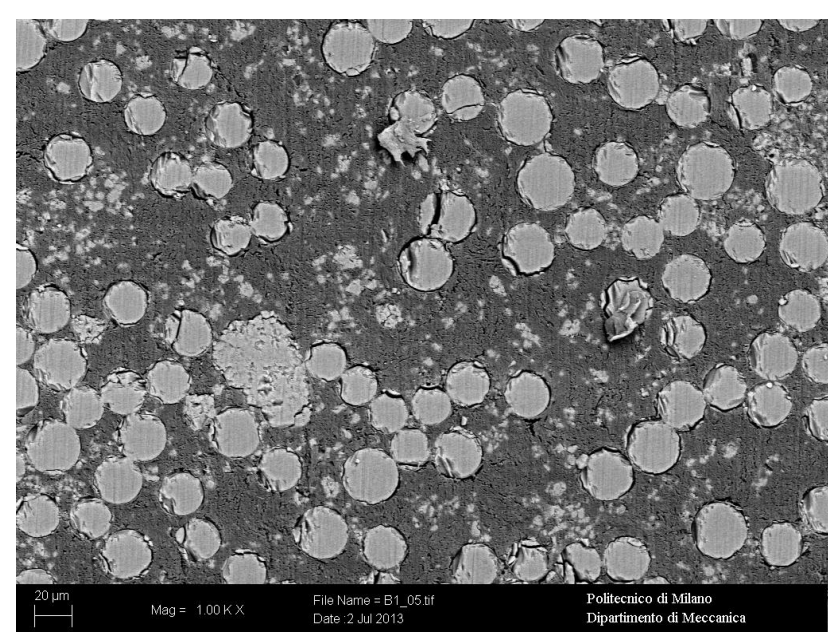

(a)

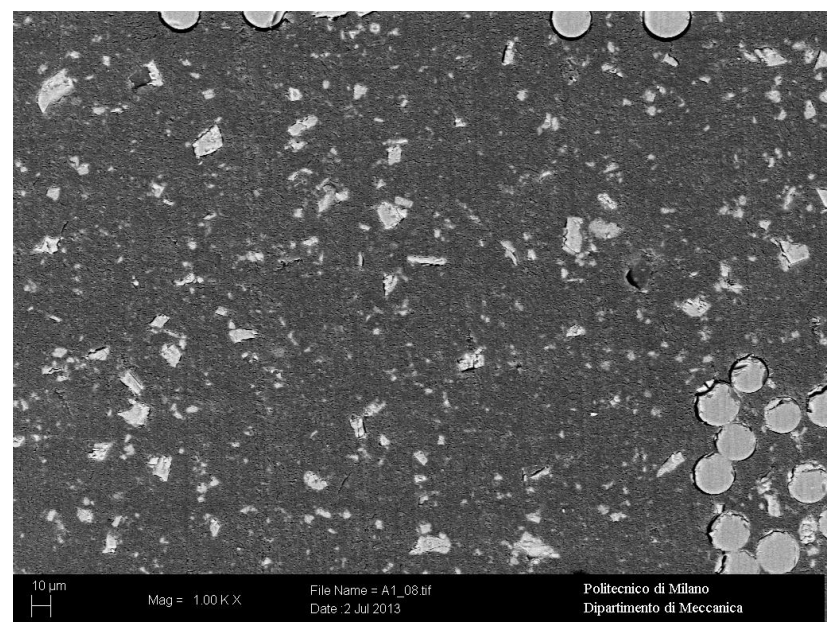

(c)

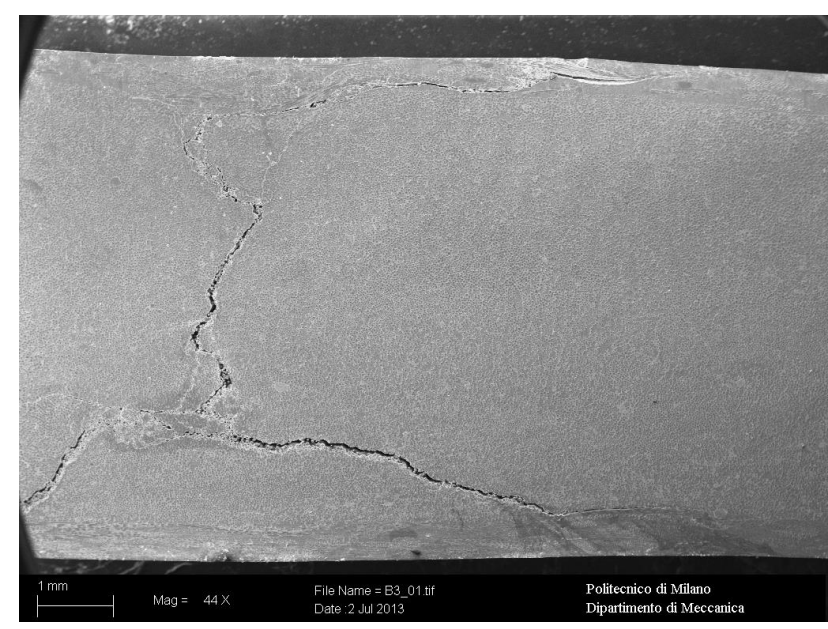

(b)

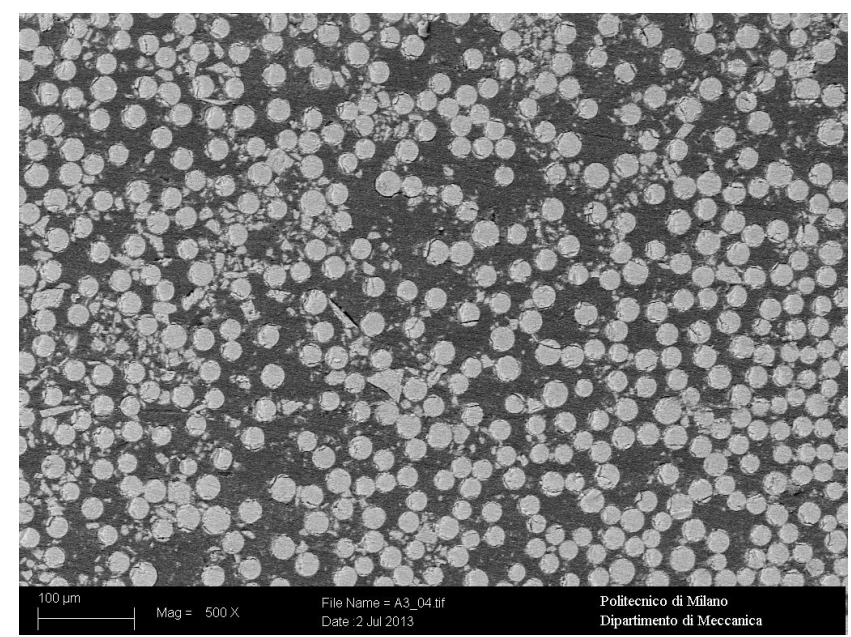

(d)

Figure 17: Scanning Electron Microscope images of cross-sections from a smooth specimen in the notch region (a) and in a far region (b) and a notched specimen in the notch region (c) and a far region (d).

Based on these observations, the tensile behavior of the material can be divided in four different phases. A first phase in which no significant $A E$ is detected shows no apparent degradation of the material; then, at about $20 \%$ of limit stress, what is thought to be matrix degradation phenomena begin and are found throughout the whole test. At $50 \%$ of the limit stress, degradation phenomena of the MAT layers start to occur (which finally lead to MAT fracture), and towards the end ( $80 \%$ of limit stress) AE that can be 
associated to fiber breakage is seen. The onset of the AE activity of cluster 1 - which is found before cluster 2 (unidirectional fiber breakage) and after cluster 3 (matrix degradation) - confirm such linkage, being the stiffness and toughness of the MAT lower than the fibers' but higher than the matrix's one.

Additionally, average AE parameters for each cluster have been calculated. The results are reported in Table 1. It can be observed that classes have some overlapping characteristics and some parameters which are distinctive, like high amplitude for cluster 2 . Besides it should be noted that the frequency parameters FMXA and FCOG are not varying much, mainly because the sensors were of the resonant type. The values of the computed parameters agree with the studies of [15], [16] which show that high-amplitude events are associated with fiber breakage and fiber pullout (cluster 2 in the present work), while low amplitude AE is associated to matrix microcracking (cluster 3). Medium amplitude events, around 50-60 dB, are linked to delamination, which is confirmed by the observation of signals of cluster 4 .

The observation of individual waveforms is also in good agreement with the findings of [20], showing similar shapes for the different waveforms associated to the corresponding failure modes (Figure 11).

Table 1: summary of AE cluster parameters

\begin{tabular}{|l|l|l|l|l|}
\hline & Cluster 1 & Cluster 2 & Cluster 3 & Cluster 4 \\
\hline $\begin{array}{l}\text { Amplitude } \\
(\mathbf{d B} \text { ae) }\end{array}$ & 54.6 & 75.2 & 47.0 & 55.3 \\
\hline Energy (eu) & $0.8^{*} 10^{\wedge} 3$ & $6.2^{*} 10^{\wedge} 5$ & $0.08^{*} 10^{\wedge} 3$ & $1.210^{\wedge} 3$ \\
\hline $\begin{array}{l}\text { Duration } \\
(\mu \mathrm{s})\end{array}$ & $3,5^{*} 10^{\wedge} 3$ & $5,5^{*} 10^{\wedge} 3$ & 54 & $3,7^{*} 10^{\wedge} 3$ \\
\hline $\begin{array}{l}\text { Risetime } \\
(\mu \mathrm{s})\end{array}$ & $0,2^{*} 10^{\wedge} 3$ & $2,4^{*} 10^{\wedge} 3$ & 19 & $3,2^{*} 10^{\wedge} 3$ \\
\hline \begin{tabular}{l} 
Counts \\
\hline
\end{tabular} & 36 & 233 & 9 & 46 \\
\hline FCOG (kHz) & 277 & 269 & 286 & 283 \\
\hline FMXA (kHz) & 266 & 260 & 263 & 261 \\
\hline
\end{tabular}

\section{Conclusions}

A technique was developed to automatically classify AE data with SOM and k-means, choosing the optimal number of clusters based on multiple quality indexes driven by a voting scheme; the technique is found to be promising in separating different AE signal sources which are related to different damage modes. On the basis of the results, the following conclusion can be drawn:

- the AE classification technique presented in this paper allowed to successfully identify the different failure modes of this type of material, providing additional information about their onset and development during the application of load;

- the classification method is quite robust to material change, and is able to classify data which was not in the training set even if the material layup changes slightly; 
- moreover, the ANN was able to identify anomalies in two specimens (the two containing a notch) with a high degree of confidence.

The ability of separating the different failure modes is of vital importance in perspective of its application to structural members. Thresholds based on classes can be defined with this aim and acceptance or rejection of a structural member can be decided by only loading it at a percent of the design load (thus reducing the risk of damaging it). The clustering method offers also a good way to eliminate the uncertainty associated to some less-dangerous AE source classes that are less active than others; this is particularly useful for the use of $A E$ as a structural health monitoring technique.

\section{Acknowledgements}

This research has been developed in the scope of a PhD scholarship provided by ETS Sistemi Industriali srl, Via Olivetti, 2, 20041 Agrate Brianza (MI), to Davide Crivelli. The authors would like also to thank the reviewers for the comments and suggestions to improve the present work.

\section{References}

1. Colombo C, Vergani L. Experimental and numerical analysis of a bus component in composite material. Comp Struct 2010;7(92).

2. Starr TF. Pultrusion for Engineers. Woodhead Publishing Ltd., 2000.

3. Jessen SM, Plumtree A. Fatigue damage accumulation in pultruded glass/polyester rods. Composites 1999;20(6).

4. Vergani L. Damage mechanisms in pultruded unidirectional fiber reinforced composites under static and fatigue loads. Fracture and Damage of Composites 2005;1.

5. Crivelli D. Acoustic emission based damage analysis of pultruded glass fiber reinforced materials. In: Youth Symposium on Experimental Solid Mechanics, 2010. p.197-200.

6. Guagliano M, Crivelli D, Marangoni D, Monici A. Fatigue Damage Analysis of Pultruded Glass Fiber Reinforced Materials with Acoustic Emission Methods. In: Proceedings of EWGAE 2010, 2010.

7. Finleyson RD. Handbook of Nondestructive Evaluation. McGraw-Hill Companies, 2003.

8. Grosse C, Ohtsu M. Acoustic Emission Testing. Springer-Verlag Berlin Heidelberg, 2008.

9. Huang M, Jiang L, Liaw PK, Brooks CR, Seeley R, Klarstrom DL. Using Acoustic Emission in Fatigue and Fracture Materials Research. JOM 1998;50(11).

10. Bourchak M, Farrow I, Bond I, Rowland C, Menan F. Acoustic emission energy as a fatigue damage parameter for CFRP composites. Int J Fatigue 2007;29(3).

11. Carpenter S, Kumosa M. An investigation of brittle fracture of composite insulator rods in an acid environment with either static or cyclic loading. J Mater Sci 2000;35(17).

12. Crivelli D, Guagliano M, Monici A. Damage Assessment in Pultruded Glass-Fiber Reinforced Materials with Acoustic Emission Testing. Struct Durability \& Health Monitor 2012;197(1). 
13. Wang X, Zhang H-P, Yan X. Classification and identification of damage mechanisms in polyethylene self-reinforced laminates by acoustic emission technique. Polym Compos 2011;32(6).

14. Gutkin R, Green CJ, Vangrattanachai S, Pinho ST, Robinson P, Curtis PT. On acoustic emission for failure investigation in CFRP: Pattern recognition and peak frequency analyses. Mech Syst Signal $\operatorname{Pr}$ 2011;25(4).

15. Kotsikos G, Evans JT, Gibson AG, Hale J. Use of acoustic emission to characterize corrosion fatigue damage accumulation in glass fiber reinforced polyester laminates. Polym Compos 1999;20.

16. Gong X, Laksimi A, Benzeggagh ML. Nouvelle approche de l'émission acoustique et son application à l'identification des mécanismes d'endommagement dans les matériaux composites. Revue des composites et des matériaux avancés 1998;8(1).

17. Haykin S. Neural networks and learning machines. Pearson International Edition, 2009.

18 Yang J, Dumont G. Classification of mixed acoustic emission signals via neural networks. Proc. SPIE 1992.

19 Calabrese L, Campanella G, Proverbio E. Use of Cluster Analysis of Acoustic Emission Signals in Evaluating Damage Severity in Concrete Structures. J. Acoust. Emission 2010;28. p 129-141

20 Godin N, Huguet S, Gaertner R. Integration of the Kohonen's self-organising map and k-means algorithm for the segmentation of the AE data collected during tensile tests on cross-ply composites. NDT\&E Int 2005;38(4), p 299-309

21. Keller T, Tirelli T, Zhou A. Tensile fatigue performance of pultruded glass fiber reinforced polymer profiles. Comp Struct 2005;68(2).

22. Kohonen T. The self-organizing map. Proceedings of the IEEE 1990;78(9).

23. Bhat $\mathrm{C}$, Bhat MR, Murthy CRL. Acoustic emission characterization of failure modes in composites with ANN. Comp Struct 2003;61.

24. Vesanto J, Alhoniemi E. Clustering of the self-organizing map. IEEE T Neural Networ 2000;11(3).

25. Gunter S, Bunke H. Validation indices for graph clustering. Pattern Recogn Lett 2003;24.

26. De Oliveira R, Marques a. T. Health monitoring of FRP using acoustic emission and artificial neural networks. Comput Struct 2008;86(3)-(5).

27. Sause MGR, Gribov A, Unwin AR, Horn S. Pattern recognition approach to identify natural clusters of acoustic emission signals. Pattern Recogn Lett 2012;33.

28. Davies DL, Bouldin LW. A cluster separation measure. IEEE T Pattern Anal 1979;1(2).

29. Rousseeuw PJ. Silhouettes: a graphical aid to the interpretation and validation of cluster analysis. J Comput Appl Math 1987;20.

30. Calinski RB, Harabasz J. A dendrite method for cluster analysis. Commun Stat 1974;3. 
31. Yosung Shim; Jiwon Chung; In-Chan Choi. A Comparison Study of Cluster Validity Indices Using a Nonhierarchical Clustering Algorithm. Comput Intell Model Control Automat 2005

32. Vesanto J, Himberg J, Alhoniemi E, Parhankangas J. Self-organizing map in Matlab: the SOM toolbox. Proceedings of the Matlab DSP Conference, 1999.

33. Pilkey WD, Pilkey DF. Peterson's Stress Concentration Factors. Hoboken, NJ, USA: John Wiley \& Sons, Inc., 2007. 